\title{
Small prime solutions of linear ternary equations
}

\author{
by \\ Hongze Li (Jinan)
}

1. Introduction. In this paper, we consider the size of small solutions of the following diophantine equation (1.1) in prime variables $p_{j}$ :

$$
a_{1} p_{1}+a_{2} p_{2}+a_{3} p_{3}=b .
$$

In particular, we estimate the numerical value of a relevant constant in the upper bound for small prime solutions of (1.1).

Let $a_{1}, a_{2}, a_{3}$ be any integers such that

$$
a_{1} a_{2} a_{3} \neq 0 \quad \text { and } \quad\left(a_{1}, a_{2}, a_{3}\right):=\operatorname{gcd}\left(a_{1}, a_{2}, a_{3}\right)=1 .
$$

Let $b$ be any integer satisfying

$$
b \equiv a_{1}+a_{2}+a_{3}(\bmod 2) \quad \text { and } \quad\left(b, a_{i}, a_{j}\right)=1 \quad \text { for } 1 \leq i<j \leq 3 .
$$

Conditions (1.2) and (1.3) are plainly necessary in our investigation, for otherwise, the equation (1.1) will either be insolvable or be reduced to fewer than three prime variables. The problem of bounds for small prime solutions $p_{1}, p_{2}, p_{3}$ of the equation (1.1) was first considered by A. Baker in connection with his now well known work [1] on the solvability of certain diophantine inequalities involving primes. Baker's investigation raised immediately the problem of obtaining the best possible upper bound for small prime solutions. In [7] the following was proved:

THEOREM 0. Assume the conditions (1.2) and (1.3). If not all $a_{1}, a_{2}, a_{3}$ are of the same sign, then there is an effective absolute constant $B>0$ such that the equation (1.1) has a prime solution $p_{1}, p_{2}, p_{3}$ satisfying

$$
\max _{1 \leq j \leq 3} p_{j} \leq 3|b|+\max \left\{3,\left|a_{1}\right|,\left|a_{2}\right|,\left|a_{3}\right|\right\}^{B} .
$$

Obviously, $B$ is the only relevant constant in (1.4). It is easy to see ([8], p. 125) that $B$ must be larger than 1 . The infimum $\mathcal{B}$ of all possible values

2000 Mathematics Subject Classification: 11P32, 11P55.

Project supported by the National Natural Science Foundation of China and the Teaching and Research Award Fund for Outstanding Young Teachers in Higher Education Institution of MOE, P.R.C. 
of the constant $B$ in (1.4) is now called the Baker constant. Plainly, the determination of $\mathcal{B}$ will completely settle the Baker problem on the bound for small prime solutions of the equation (1.1).

It was shown in [7] that Theorem 0 contains the well known Linnik Theorem on the smallest prime in an arithmetic progression, namely, for any positive integers $l, q$ with $l \leq q$ and $(l, q)=1$, the smallest prime $P(l, q)$ in the arithmetic progression $l+k q$ satisfies $P(l, q) \ll q^{L}$ where $L$ is an absolute positive constant. The infimum $\mathcal{L}$ of all values of $L$ is called the Linnik constant. It was shown in [8] that $\mathcal{B} \geq \mathcal{L}$. Many authors (see Table 1 in [5]) investigated the numerical bounds for $\mathcal{L}$. The first numerical result for $\mathcal{B}$ was obtained by Choi [2]: $\mathcal{B} \leq 4190$. In [9] Liu and Wang proved that $\mathcal{B} \leq 45$. In [3] Choi, Liu and Tsang proved that $\mathcal{B} \leq 4$ under the Generalized Riemann Hypothesis. In the present paper we prove that $\mathcal{B} \leq 38$ :

TheOREM 1. Assume the conditions (1.2) and (1.3). If not all $a_{1}, a_{2}$, $a_{3}$ are of the same sign, then there is an absolute constant $C>0$ such that the equation (1.1) has a prime solution $p_{1}, p_{2}, p_{3}$ satisfying

$$
\max _{1 \leq j \leq 3}\left|a_{j}\right| p_{j} \leq C \max \left\{|b|,\left(\max \left\{\left|a_{1}\right|,\left|a_{2}\right|,\left|a_{3}\right|\right\}\right)^{38}\right\} .
$$

That is, $\mathcal{B} \leq 38$.

It should be noted that the constant 38 can be reduced a little by the method of [3].

2. Some lemmas. As usual, let $\chi(\bmod q)$ and $\chi_{0}(\bmod q)$ denote a character and the principal character modulo $q$ respectively. $L(s, \chi)$ denotes a Dirichlet $L$-function, and $\varepsilon$ and $\varepsilon_{j}$ are small positive numbers. Let

$$
\Pi(s):=\prod_{q \leq Q} \prod_{\chi(\bmod q)}^{*} L(s, \chi) .
$$

We consider the zero-free regions of the function $\Pi(s)$ in the region $|\operatorname{Im}(s)| \leq$ $C, 1 / 2 \leq \operatorname{Re}(s) \leq 1$, where $Q$ is a given sufficiently large positive number, $C$ is any positive constant, and the $*$ indicates that the product $\Pi^{*}$ is over all primitive characters $\chi(\bmod q)$. We put

$$
\mathcal{L}:=\log Q .
$$

We begin by proving that for sufficiently large $Q$ there exists an absolute positive constant $k$ such that none of the functions $L(s, \chi)$ with characters $\chi(\bmod q), q \leq Q$, have zeros in the rectangles

$$
1-10 / \mathcal{L} \leq \sigma \leq 1, \quad 10^{k} C<|t| \leq 10^{k+1} C .
$$


As in Lemma 6.1 of [3], this is a consequence of the estimate

$$
\sum_{q \leq Q} \sum_{\chi(\bmod q)} N(\sigma, T, \chi) \ll_{\varepsilon}\left(Q^{2} T\right)^{(2+\varepsilon)(1-\sigma)} \quad(4 / 5 \leq \sigma \leq 1, T \geq 1, \varepsilon>0)
$$

due to Jutila. Let $\sigma=1-10 / \mathcal{L}$. Jutila's bound implies that

$$
\sum_{q \leq Q} \sum_{\chi(\bmod q)} N(\sigma, \mathcal{L}, \chi) \ll_{\varepsilon}\left(Q^{2} \mathcal{L}\right)^{(2+\varepsilon)(1-\sigma)} \ll\left(Q^{2} \mathcal{L}\right)^{3(1-\sigma)}=O(1)
$$

providing that $Q$ is sufficiently large. Similarly to Lemma 6.1 of [3], on choosing an appropriate $k$, we deduce the conclusion.

We proceed to number certain of the characters $\chi(\bmod q)$ and zeros $\varrho=\beta+i \gamma$ of $L(s, \chi)$ as follows. Let $\varrho$ denote any zero of $\Pi(s)$ in the rectangle

$$
R=\left\{s=\sigma+i t: 1-10 \mathcal{L}^{-1} \leq \sigma \leq 1,|t| \leq 10^{k} C\right\} .
$$

Denote by $\varrho_{1}$ one of the above zeros for which $\beta$ is maximal, and let $\chi_{1}$ be a corresponding primitive character in $(2.1)$ such that $L\left(\varrho_{1}, \chi_{1}\right)=0$. Now, remove $L\left(s, \chi_{1}\right)$ and $L\left(s, \bar{\chi}_{1}\right)$ from $(2.1)$, and choose $\varrho_{2}$ to be one of the zeros of $\Pi(s)\left(L\left(s, \chi_{1}\right) L\left(s, \bar{\chi}_{1}\right)\right)^{-1}$ in $R$, for which $\beta$ is maximal. We take $\chi_{2}$ to be a primitive character in $(2.1)$ for which $L\left(\varrho_{2}, \chi_{2}\right)=0$. Then by arguments similar to [5] we see that if a primitive character $\chi \neq \chi_{1}, \bar{\chi}_{1}$, then every zero $\varrho$ of $L(s, \chi)$ satisfies

$$
\operatorname{Re}(\varrho) \leq \operatorname{Re}\left(\varrho_{2}\right) \quad \text { or } \quad|\operatorname{Im}(\varrho)|>10^{k+1} C .
$$

For convenience of notation we shall set

$$
\varrho_{k}=\beta_{k}+i \gamma_{k}, \quad \beta_{k}=1-\mathcal{L}^{-1} \lambda_{k} .
$$

Suppose that $L\left(s, \chi_{1}\right)$ has a zero $\varrho^{\prime} \neq \varrho_{1}$ in the rectangle $R$, given by $(2.3)$ (or a repeated zero $\varrho^{\prime}=\varrho_{1}$ ); in case $\chi_{1}$ is real and $\varrho_{1}$ is complex we choose such a zero $\varrho^{\prime} \neq \varrho_{1}$ with $\operatorname{Re}\left(\varrho^{\prime}\right)$ maximal, and put

$$
\varrho^{\prime}=\beta^{\prime}+i \gamma^{\prime}, \quad \beta^{\prime}=1-\mathcal{L}^{-1} \lambda^{\prime}
$$

in analogy to the above.

Lemma 1. For any constant $C>0$, there exists a $K(C)>0$ depending on $C$ only such that if $Q \geq K(C)$, then the function $\Pi(s)$ defined by (2.1) has at most one zero in the region $\sigma \geq 1-0.364 / \mathcal{L},|t| \leq 10^{k} C$. Such a zero $\widetilde{\beta}$, if it exists, is real and simple, and corresponds to a non-principal, real, primitive character $\tilde{\chi}$ to a modulus $\widetilde{r} \leq Q$. $\widetilde{\beta}$ is called the Siegel zero or the exceptional zero.

This is Proposition 2.3 of [9].

Lemma 2. For any constant $C>0$, there exists a $K(C)>0$ depending on $C$ only such that if $Q \geq K(C)$, then the function $\Pi(s)$ defined by (2.1) 
has at most two zeros in the region $\sigma \geq 1-0.504 / \mathcal{L},|t| \leq 10^{k} C$. Moreover, the bounds in Table 1 can be applied.

Table 1. The lower bound for $\lambda_{2}$

\begin{tabular}{ll|lc}
\hline$\lambda_{1}$ & $\lambda_{2}$ & $\lambda_{1}$ & $\lambda_{2}$ \\
\hline 0.348 & 0.587 & 0.45 & 0.530 \\
0.36 & 0.578 & 0.48 & 0.516 \\
0.40 & 0.555 & 0.504 & 0.504 \\
\hline
\end{tabular}

This is Lemma 2.5 of [9].

Lemma 3. If the exceptional zero $\widetilde{\beta}$ in Lemma 1 does indeed exist, then for any constant $c$ with $0<c<1$ and for any small $\varepsilon>0$ there is $a K(c, \varepsilon)>0$ depending on $c$ and $\varepsilon$ only such that for any zero $\varrho=\beta+i \gamma \neq$ $\widetilde{\beta}($ corresponding to $\chi(\bmod q))$ of the function $\Pi(s)$ defined by $(2.1)$ we have

$$
\beta \leq 1-\min \left\{\frac{c}{6}, \frac{(1-c)(8 / 9-\varepsilon)}{\log ([\widetilde{r}, q]|\gamma|)} \log \left(\frac{(1-c)(8 / 9-\varepsilon)}{(1-\widetilde{\beta}) \log ([\widetilde{r}, q]|\gamma|)}\right)\right\},
$$

if $[\widetilde{r}, q]|\gamma|>K(c, \varepsilon)$. Moreover, for any positive $\varepsilon$ there exists a constant $c(\varepsilon)>0$ depending on $\varepsilon$ only such that

$$
1-0.364 / \mathcal{L} \leq \widetilde{\beta} \leq 1-c(\varepsilon) \widetilde{r}^{-\varepsilon} .
$$

Proof. By Lemma 2.4 of [6], if $\chi$ is a non-principal character modulo $q$, and $\phi=3 / 8$, then for any $\varepsilon>0$ there exists a $\delta=\delta(\varepsilon)>0$ such that

$$
-\operatorname{Re}\left(\frac{L^{\prime}}{L}(s, \chi)\right) \leq-\sum_{|1+i t-\varrho| \leq \delta} \operatorname{Re}\left(\frac{1}{s-\varrho}\right)+\left(\frac{\phi}{2}+\varepsilon\right) \mathcal{L}
$$

uniformly for

$$
1+\frac{1}{\mathcal{L} \log \mathcal{L}} \leq \sigma \leq 1+\frac{\log \mathcal{L}}{\mathcal{L}}
$$

providing that $q$ is sufficiently large, where $\mathcal{L}=\log q(|t|+2)$. As in Lemmas $8.1,8.5,8.6$ of [5], we can get Principle 2 of [5], p. 266, with $c_{2} \leq 8 / 9$ providing that $q$ is large enough and $\delta$ is small enough. Similarly to Lemma 2.6 of [9], the assertion follows.

Let

$$
\begin{gathered}
\alpha=1-\lambda / \mathcal{L} \quad \text { for } 0.364 \leq \lambda \leq \log \log \mathcal{L}, \\
D:=\{s=\sigma+i t: \alpha \leq \sigma \leq 1-0.364 / \mathcal{L},|t| \leq C\}, \\
N(\chi, \alpha, C):=\text { number of zeros of } L(s, \chi) \text { in } D, \\
N^{*}(\alpha, Q, C):=\sum_{q \leq Q} \sum_{\chi(\bmod q)}^{*} N(\chi, \alpha, C),
\end{gathered}
$$

where $\sum^{*}$ denotes that the sum is over primitive characters. 
Lemma 4. For any absolute constant $C>0$, let $\alpha=1-\lambda / \mathcal{L}$. Then for $Q \geq K(C)$ which is a positive constant depending on $C$ only, we have

$$
N^{*}(\alpha, Q, C) \leq \begin{cases}8.86706 C_{1}(\lambda) e^{4.31403 \lambda}, & 0.504<\lambda \leq 0.696, \\ 26.93 C_{2}(\lambda) e^{4.28374 \lambda}, & 0.696<\lambda \leq 1, \\ 50.36 C_{3}(\lambda) e^{3.753506 \lambda}, & 1<\lambda \leq 2, \\ 167.67 C_{4}(\lambda) e^{3.116796 \lambda}, & 2<\lambda \leq 6, \\ 42.54(\lambda+35.385) C_{5}(\lambda) e^{2.87538 \lambda}, & 6<\lambda \leq \log \log \mathcal{L},\end{cases}
$$

where

$$
\begin{aligned}
& C_{1}(\lambda)=\lambda^{-1}\left(1-e^{-4.31403 \lambda} \frac{e^{3.15402 \lambda}-e^{2.32002 \lambda}}{0.834 \lambda}\right), \\
& C_{2}(\lambda)=\lambda^{-1}\left(1-e^{-4.28374 \lambda} \frac{e^{3.19253 \lambda}-e^{2.42653 \lambda}}{0.766 \lambda}\right), \\
& C_{3}(\lambda)=\lambda^{-1}\left(1-e^{-3.753506 \lambda} \frac{e^{2.747904 \lambda}-e^{2.160104 \lambda}}{0.58 \lambda}\right), \\
& C_{4}(\lambda)=\lambda^{-1}\left(1-e^{-3.116796 \lambda} \frac{e^{2.223794 \lambda}-e^{1.869794 \lambda}}{0.354 \lambda}\right), \\
& C_{5}(\lambda)=\lambda^{-1}\left(1-e^{-2.87538 \lambda} \frac{e^{2.07176 \lambda}-e^{1.92136 \lambda}}{0.1504 \lambda}\right) .
\end{aligned}
$$

This is Lemma 3.1 of [9].

3. The circle method. From now on, we let $N$ be a sufficiently large positive number, and let

$$
\theta:=1 /\left(38 / 3-20 \varepsilon_{1}\right), \quad Q:=N^{\theta}, \quad T:=Q^{5 / 2+3 \varepsilon_{1}}, \quad \tau:=N^{-1} Q^{1+\varepsilon_{1}},
$$

where $\varepsilon_{1}$ is a fixed sufficiently small positive number. For $1 \leq j \leq 3$, let

$$
\begin{gathered}
N_{j}:=N\left|a_{j}\right|^{-1}, \quad N_{j}^{\prime}:=N\left(4\left|a_{j}\right|\right)^{-1}, \\
B:=\max \left\{\left|a_{1}\right|,\left|a_{2}\right|,\left|a_{3}\right|\right\} .
\end{gathered}
$$

We always assume

$$
B^{3+3 \varepsilon_{1}} \ll Q .
$$

Denote by $\Lambda(n)$ the von Mangoldt function, and define, for any real $y$,

$$
\begin{gathered}
S_{j}(y):=\sum_{N_{j}^{\prime}<n \leq N_{j}} \Lambda(n) e\left(a_{j} n y\right), \\
I(b):=\sum_{\left(n_{1}, n_{2}, n_{3}\right)} \Lambda\left(n_{1}\right) \Lambda\left(n_{2}\right) \Lambda\left(n_{3}\right),
\end{gathered}
$$

where $\sum_{\left(n_{1}, n_{2}, n_{3}\right)}$ denotes the summation over all triples $\left(n_{1}, n_{2}, n_{3}\right)$ satisfying $\sum_{1 \leq j \leq 3} a_{j} n_{j}=b$ and $N_{j}^{\prime}<n_{j} \leq N_{j}, 1 \leq j \leq 3$. For any integers 
$h$ and $q$ with $1 \leq h \leq q \leq Q$ and $(h, q)=1$, let $m(h, q)$ be the interval $[(h-\tau) / q,(h+\tau) / q]$. Let $\mathcal{M}$ be the union of these mutually disjoint intervals and $\mathcal{M}^{\prime}$ be the complement of $\mathcal{M}$ in $[\tau, 1+\tau]$. By (3.5) and (3.6) we have

$$
I(b)=\left\{\int_{\mathcal{M}}+\int_{\mathcal{M}^{\prime}}\right\} e(-b x) \prod_{j=1}^{3} S_{j}(x) d x=: I_{1}(b)+I_{2}(b), \quad \text { say, }
$$

where $I_{1}(b)$ and $I_{2}(b)$ are the integrals on $\mathcal{M}$ and $\mathcal{M}^{\prime}$ respectively. For any integer $n$ and any character $\chi(\bmod q)$, denote the Gaussian sum by

$$
G(n, \chi):=\sum_{l=1}^{q} \chi(l) e(n l / q) \text { and let } G(n, q):=G\left(n, \chi_{0}\right) .
$$

If $x \in m(h, q)$, write

$$
x=h / q+\eta, \quad(h, q)=1, \quad|\eta| \leq \tau q^{-1} .
$$

From now on, we put $L:=\log N$. For any real $\eta$ and any $\chi(\bmod q)$ with $q \leq Q, 1 \leq j \leq 3$, let

$$
\begin{gathered}
I_{j}(\eta):=\int_{N_{j}^{\prime}}^{N_{j}} e\left(a_{j} \eta x\right) d x, \quad \widetilde{I}_{j}(\eta):=\int_{N_{j}^{\prime}}^{N_{j}} x^{\widetilde{\beta}-1} e\left(a_{j} \eta x\right) d x, \\
I_{j}(\chi, \eta):=\int_{N_{j}^{\prime}}^{N_{j}} e\left(a_{j} \eta x\right) \sum_{|\gamma| \leq T}^{\prime} x^{\varrho-1} d x,
\end{gathered}
$$

where $\widetilde{\beta}$ is the possible Siegel zero in Lemma 1 and the corresponding character is $\tilde{\chi}(\bmod \widetilde{r}), \sum_{|\gamma| \leq T}^{\prime}$ denotes the summation over all zeros $\varrho=\beta+i \gamma$ of $L(s, \chi)$ satisfying $\varrho \neq \widetilde{\beta},|\gamma| \leq T$ and $1 / 2 \leq \beta \leq 1$.

Lemma 5. Let

$$
\begin{gathered}
\mathcal{G}_{j}(h, q, \eta):=\sum_{\chi(\bmod q)} G\left(a_{j} h, \bar{\chi}\right) I_{j}(\chi, \eta), \\
H_{j}(h, q, \eta):=G\left(a_{j} h, q\right) I_{j}(\eta)-\delta(q) G\left(a_{j} h, \widetilde{\chi} \chi_{0}\right) \widetilde{I}_{j}(\eta)-\mathcal{G}_{j}(h, q, \eta),
\end{gathered}
$$
where $\delta(q)=1$ if $\widetilde{r} \mid q$ and $\delta(q)=0$ otherwise. Then for any $x \in m(h, q)$,

$$
S_{j}(x)=\varphi(q)^{-1} H_{j}(h, q, \eta)+O\left((1+N|\eta|) N_{j} q^{1 / 2} T^{-1} L^{2}\right) .
$$

This is Lemma 4.2 of [9].

Lemma 6. For any $x \geq 1$ and $y \geq 2$, let

$$
N(\alpha, x, y):=\sum_{q \leq x} \sum_{\chi(\bmod q)}^{*} \sum_{\substack{\varrho=\beta+i \gamma \\|\gamma| \leq y, \beta \geq \alpha}} 1,
$$


where $\varrho=\beta+i \gamma$ is any non-trivial zero of $L(s, \chi)$. Then

$$
\begin{array}{ll}
N(\alpha, x, y) \ll\left(x^{2} y^{6 / 5}\right)^{(20 / 9+\varepsilon)(1-\alpha)} & \text { for } 1 / 2 \leq \alpha \leq 1, \\
N(\alpha, x, y) \ll\left(x^{2} y\right)^{(2+\varepsilon)(1-\alpha)} & \text { for } 11 / 14 \leq \alpha \leq 1 .
\end{array}
$$

See Theorems 1 and 2 of [4].

LEMMA 7. Under the notations and conditions of (3.1) to (3.4), we have for $1 \leq j \leq 3$,

$$
\Sigma_{1}:=\sum_{q \leq Q} \sum_{\chi(\bmod q)}^{*} \sum_{|\gamma| \leq T} N_{j}^{\beta-1} \ll 1 .
$$

Proof. In view of $Q \geq B^{3}$ and $\theta=1 /\left(38 / 3-20 \varepsilon_{1}\right)$, we have for $1 \leq j \leq 3$,

$$
N_{j} \geq Q^{38 / 3-20 \varepsilon_{1}-1 / 3} \text {. }
$$

As in Lemma 4.5 of [9], note that $T=Q^{2.5+3 \varepsilon_{1}}$; by Lemma 6 , the assertion follows.

Lemma 8. Under the conditions of Lemma 7 , for $1 \leq j \leq 3$ we have

$$
S_{1, j}:=\sum_{q \leq Q} \sum_{\chi(\bmod q)}^{*} \sum_{|\gamma| \leq T}\left(\int_{-\tau / q}^{\tau / q}\left|\int_{N_{j}^{\prime}}^{N_{j}} x^{\varrho-1} e\left(a_{j} x \eta\right) d x\right|^{2} d \eta\right)^{1 / 2} \ll N^{1 / 2}\left|a_{j}\right|^{-1} .
$$

Proof. Similarly to Lemma 4.6 of [9], this follows from Lemma 7.

Lemma 9. Let $I_{1}(b)$ be defined as in (3.7). Under the assumptions of Lemma 7, we have

$$
I_{1}(b)=\sum_{q \leq Q} \varphi^{-3}(q) \sum_{h=1}^{q} e\left(-\frac{b}{q} h\right) \int_{-\tau / q}^{\tau / q} e(-b \eta) \prod_{j=1}^{3} H_{j}(h, q, \eta) d \eta+O\left(\Omega_{1}\right),
$$

where $\sum_{h=1}^{q \prime}$ is the summation over all $1 \leq h \leq q,(h, q)=1$, and

$$
\Omega_{1}:=N^{2} Q^{2.5+\varepsilon_{1}}\left|a_{1} a_{2} a_{3}\right|^{-1} T^{-1} L^{2} .
$$

Proof. As in Lemma 4.7 of [9], the assertion follows from Lemma 8.

4. The estimation of $M_{1}$ and $M_{3}$. Multiplying out the product $\prod_{j=1}^{3} H_{j}(h, q, \eta)$ in Lemma 9 , we get 27 terms (if $\widetilde{\beta}$ exists). They are grouped into the following three categories:

- $\mathcal{J}_{1}$ : the term $\prod_{j=1}^{3} G\left(a_{j} h, q\right) I_{j}(\eta)$;

- $\mathcal{J}_{2}$ : 19 terms (if $\widetilde{\beta}$ exists), each has at least one $\mathcal{G}_{j}(h, q, \eta)$ as a factor;

- $\mathcal{J}_{3}$ : the 7 terms remaining (if $\widetilde{\beta}$ exists). 
For $i=1,2,3$, define

$$
\begin{aligned}
M_{i}:= & \sum_{q \leq Q} \varphi^{-3}(q) \sum_{h=1}^{q} e\left(-\frac{b}{q} h\right) \\
& \left.\times \int_{-\tau / q}^{\tau / q} e(-b \eta) \text { sum of the terms in } \mathcal{J}_{i}\right\} d \eta .
\end{aligned}
$$

Then by Lemma 9 we get

$$
I_{1}(b)=M_{1}+M_{2}+M_{3}+O\left(\Omega_{1}\right),
$$

if one assumes (3.4). For the estimation of $M_{1}$ and $M_{3}$, we need the following notations. For any positive integer $q$, define

$$
A(q):=\varphi^{-3}(q) \sum_{h=1}^{q} e\left(-\frac{b}{q} h\right) \prod_{j=1}^{3} G\left(a_{j} h, q\right) .
$$

By Lemma 4.1 of $[7], A(q)$ is multiplicative. For any prime $p$, put

$$
s(p):=1+A(p) .
$$

Let $r_{1}, r_{2}, r_{3}$ be any positive integers and denote by $\left[r_{1}, r_{2}, r_{3}\right]$ the least common multiple of $r_{1}, r_{2}, r_{3}$. For any primitive characters $\chi_{j}\left(\bmod r_{j}\right)$ $(1 \leq j \leq 3)$ and $\left[r_{1}, r_{2}, r_{3}\right] \mid q$, define

$$
Z(q):=Z\left(q ; \chi_{1}, \chi_{2}, \chi_{3}\right)=\sum_{h=1}^{q} e\left(-\frac{b}{q} h\right) \prod_{j=1}^{3} G\left(a_{j} h, \chi_{j} \chi_{0}\right),
$$

where $\chi_{0}$ is the principal character modulo $q$, and

$$
\mathcal{D}:=\left\{\left(x_{1}, x_{2}\right):\left(4\left|a_{j}\right|\right)^{-1} \leq x_{j} \leq\left|a_{j}\right|^{-1}, j=1,2,3\right\},
$$

where $x_{3}=a_{3}^{-1}\left(b N^{-1}-a_{1} x_{1}-a_{2} x_{2}\right)$.

Lemma 10. Let $\chi$ be a character modulo $q$, induced by a primitive character $\chi^{*}$ modulo $q^{*}, q=q_{1} q_{2},\left(q_{2}, q^{*}\right)=1, p\left|q_{1} \Rightarrow p\right| q^{*}$. Then $G(m, \chi)$

$$
=\bar{\chi}^{*}\left(\frac{m}{(m, q)}\right) \chi^{*}\left(\frac{q}{q^{*}(m, q)}\right) \mu\left(\frac{q}{q^{*}(m, q)}\right) \varphi(q) \varphi^{-1}\left(\frac{q}{(m, q)}\right) G\left(1, \chi^{*}\right)
$$

if $q^{*}=q_{1} /\left(m, q_{1}\right)$; otherwise $G(m, \chi)=0$.

This is Lemma 1.5 of [10].

Lemma 11. Let $Z(q)$ and $s(p)$ be defined as in (4.5) and (4.4) respectively, and let $r=\left[r_{1}, r_{2}, r_{3}\right]$. Then under the conditions of (1.2) and (1.3), 
we have

$$
\prod_{p} s(p) \gg 1
$$

and

$$
\sum_{\substack{q \leq Q \\ r \mid q}} \varphi^{-3}(q)|Z(q)| \leq 2.140782 \prod_{p} s(p) .
$$

In particular, if two of $r_{1}, r_{2}, r_{3}$ are 1 , for example, $r_{1}=r_{2}=1, r_{3}=r$, then

$$
\sum_{\substack{q \leq Q \\ r \mid q}} \varphi^{-3}(q)|Z(q)| \ll \frac{r}{\varphi(r) \varphi\left(\frac{r}{\left(a_{1}, r\right)}\right) \varphi\left(\frac{r}{\left(a_{2}, r\right)}\right)} \prod_{p} s(p) .
$$

If one of $r_{1}, r_{2}, r_{3}$ is 1 , for example, $r_{1}=1,\left[r_{2}, r_{3}\right]=r$, then

$$
\sum_{\substack{q \leq Q \\ r \mid q}} \varphi^{-3}(q)|Z(q)| \ll Q^{\varepsilon} \frac{\left(a_{1}, r\right)}{\sqrt{r}} \prod_{p} s(p) .
$$

Proof. (4.7) and (4.8) are Lemma 5.2 of [9]. Now we consider (4.9). By the proof of Lemma 4.6 of [7] we have

$$
\begin{aligned}
\sum_{\substack{q \leq Q \\
r \mid q}} \varphi^{-3}(q)|Z(q)| & =\varphi^{-3}(r)|Z(r)| \sum_{\substack{q \leq Q / r \\
(q, r)=1}}|A(q)| \\
& \ll \varphi^{-3}(r)|Z(r)| \prod_{p \nmid r}(1+|A(p)|) .
\end{aligned}
$$

If $r_{1}=r_{2}=1, r_{3}=r$, then by $(4.5)$, for $(h, r)=1$ we have

$$
Z(r)=G\left(a_{1}, \chi_{0}\right) G\left(a_{2}, \chi_{0}\right) G\left(a_{3}, \chi_{3}\right) G\left(-b, \bar{\chi}_{3}\right) .
$$

By Lemma 1.2 of [10] we have

$$
\left|G\left(a_{1}, \chi_{0}\right)\right| \leq \varphi(r) \varphi^{-1}\left(\frac{r}{\left(a_{1}, r\right)}\right), \quad\left|G\left(a_{2}, \chi_{0}\right)\right| \leq \varphi(r) \varphi^{-1}\left(\frac{r}{\left(a_{2}, r\right)}\right) .
$$

By Lemma 1.6 of [10] we have

$$
\left|G\left(a_{3}, \chi_{3}\right)\right| \leq \sqrt{r}, \quad\left|G\left(-b, \bar{\chi}_{3}\right)\right| \leq \sqrt{r} .
$$

Hence by (4.11) and (4.12),

$$
\varphi^{-3}(r)|Z(r)| \ll \frac{r}{\varphi(r) \varphi\left(\frac{r}{\left(a_{1}, r\right)}\right) \varphi\left(\frac{r}{\left(a_{2}, r\right)}\right)} .
$$

As in Lemma 5.2 of [9], (4.9) follows.

For $(4.10)$, if $r_{1}=1,\left[r_{2}, r_{3}\right]=r$, then by (4.5) we have

$$
Z(r)=G\left(a_{1}, \chi_{0}\right) G\left(a_{2}, \chi_{2} \chi_{0}\right) G\left(a_{3}, \chi_{3} \chi_{0}\right) G\left(-b, \bar{\chi}_{2} \bar{\chi}_{3} \chi_{0}\right) .
$$


Let $\left(r_{2}, r_{3}\right)=r_{4}, r_{2}=r_{4} r_{2}^{\prime}, r_{3}=r_{4} r_{3}^{\prime}$. Then $\left(r_{2}^{\prime}, r_{3}^{\prime}\right)=1$. Let $r_{2}^{\prime}=r_{5} r_{5}^{\prime}$, $\left(r_{5}, r_{5}^{\prime}\right)=1,\left(r_{4}, r_{5}^{\prime}\right)=1, p\left|r_{5} \Rightarrow p\right| r_{4}, r_{3}^{\prime}=r_{6} r_{6}^{\prime},\left(r_{6}, r_{6}^{\prime}\right)=1,\left(r_{4}, r_{6}^{\prime}\right)=1$, $p\left|r_{6} \Rightarrow p\right| r_{4}$. Then $r=r_{4} r_{5} r_{5}^{\prime} r_{6} r_{6}^{\prime}$. By Lemma 1.2 of [10] we have

$$
\left|G\left(a_{1}, \chi_{0}\right)\right| \leq \varphi(r) \varphi^{-1}\left(\frac{r}{\left(a_{1}, r\right)}\right) .
$$

By Lemma 10, if $\left(a_{2}, r_{2} r_{6}\right)=r_{6}$, then

$$
\left|G\left(a_{2}, \chi_{2} \chi_{0}\right)\right| \leq \varphi(r) \varphi^{-1}\left(\frac{r}{\left(a_{2}, r\right)}\right) \sqrt{r_{2}},
$$

otherwise $\left|G\left(a_{2}, \chi_{2} \chi_{0}\right)\right|=0$. Similarly, if $\left(a_{3}, r_{3} r_{5}\right)=r_{5}$, then

$$
\left|G\left(a_{3}, \chi_{3} \chi_{0}\right)\right| \leq \varphi(r) \varphi^{-1}\left(\frac{r}{\left(a_{3}, r\right)}\right) \sqrt{r_{3}},
$$

otherwise $\left|G\left(a_{3}, \chi_{3} \chi_{0}\right)\right|=0$. We may put

$$
\bar{\chi}_{2}=\bar{\chi}_{4}^{(1)} \bar{\chi}_{5} \bar{\chi}_{5}^{\prime}, \quad \bar{\chi}_{3}=\bar{\chi}_{4}^{(2)} \bar{\chi}_{6} \bar{\chi}_{6}^{\prime},
$$

where $\chi_{i} \bmod r_{i}$ and $\chi_{i}^{\prime} \bmod r_{i}^{\prime}$ are primitive; thus we have

$$
\bar{\chi}_{2} \bar{\chi}_{3} \chi_{0}=\bar{\chi}_{4} \bar{\chi}_{5} \bar{\chi}_{6} \bar{\chi}_{5}^{\prime} \bar{\chi}_{6}^{\prime},
$$

where $\chi_{4}=\chi_{4}^{(1)} \chi_{4}^{(2)}$. Hence by Lemma 10 we have

$$
\begin{aligned}
& \left|G\left(-b, \bar{\chi}_{2} \bar{\chi}_{3} \chi_{0}\right)\right| \\
& \leq\left|G\left(-b, \bar{\chi}_{4} \bar{\chi}_{5} \bar{\chi}_{6}\right) G\left(-b, \bar{\chi}_{5}^{\prime}\right) G\left(-b, \bar{\chi}_{6}^{\prime}\right)\right| \\
& \leq \sqrt{r_{5}^{\prime}} \sqrt{r_{6}^{\prime}}\left|G\left(-b, \bar{\chi}_{4} \bar{\chi}_{5} \bar{\chi}_{6}\right)\right| \\
& \leq \sqrt{r_{5}^{\prime} r_{6}^{\prime}} \varphi\left(r_{4} r_{5} r_{6}\right) \varphi^{-1}\left(\frac{r_{4} r_{5} r_{6}}{\left(b, r_{4} r_{5} r_{6}\right)}\right) \sqrt{\frac{r_{4} r_{5} r_{6}}{\left(b, r_{4} r_{5} r_{6}\right)}} \leq \sqrt{r_{5}^{\prime} r_{6}^{\prime}} r_{4} r_{5} r_{6} .
\end{aligned}
$$

Hence

$$
\varphi^{-3}(r)|Z(r)| \leq \frac{r^{2} \sqrt{r_{5} r_{6}}}{\varphi^{2}(r) \varphi\left(\frac{r}{\left(a_{1}, r\right)}\right)} \leq Q^{\varepsilon^{2}} \frac{\left(a_{1}, r\right) \sqrt{r_{5} r_{6}}}{r} \leq Q^{\varepsilon} \frac{\left(a_{1}, r\right)}{\sqrt{r}} .
$$

So (4.10) follows.

Lemma 12. Let $M_{1}$ be defined as in (4.1). If not all the $a_{j}$ 's are of the same sign and $N \geq 3|b|$, then

$$
M_{1}=M_{0}+O\left(\Omega_{2}\right),
$$

where

$$
M_{0}:=N^{2}\left|a_{3}\right|^{-1}\left(\prod_{p} s(p)\right) \int_{\mathcal{D}} d x_{1} d x_{2}
$$


with $\mathcal{D}$ given by (4.6), and

$$
M_{0} \gg N^{2}\left|a_{1} a_{2} a_{3}\right|^{-1} \prod_{p} s(p), \quad \Omega_{2}:=N^{2} Q^{-1+\varepsilon_{1} / 4}\left|a_{1} a_{2} a_{3}\right|^{-1} .
$$

See Lemmas 5.3 and 7.4 of [9].

LEMma 13. If the exceptional zero $\widetilde{\beta}$ defined as in Lemma 1 exists, put

$$
\omega:=(1-\widetilde{\beta}) \mathcal{L} .
$$

Then

$$
M_{1}+M_{3} \geq \begin{cases}20 \omega^{3} M_{0}+O\left(\widetilde{r} \Omega_{2}+N^{2} Q^{-1-\varepsilon_{1}}\right) & \text { if } 0.34<\omega \leq 0.364, \\ 24.3 \omega^{3} M_{0}+O\left(\widetilde{r} \Omega_{2}+N^{2} Q^{-1-\varepsilon_{1}}\right) & \text { if } 0.326<\omega \leq 0.34, \\ 27.3 \omega^{3} M_{0}+O\left(\widetilde{r} \Omega_{2}+N^{2} Q^{-1-\varepsilon_{1}}\right) & \text { if } 0.306<\omega \leq 0.326, \\ 32.55 \omega^{3} M_{0}+O\left(\widetilde{r} \Omega_{2}+N^{2} Q^{-1-\varepsilon_{1}}\right) & \text { if } 0.23<\omega \leq 0.306, \\ 68.5 \omega^{3} M_{0}+O\left(\widetilde{r} \Omega_{2}+N^{2} Q^{-1-\varepsilon_{1}}\right) & \text { if } 0.2<\omega \leq 0.23, \\ 95.7 \omega^{3} M_{0}+O\left(\widetilde{r} \Omega_{2}+N^{2} Q^{-1-\varepsilon_{1}}\right) & \text { if } 0.1<\omega \leq 0.2, \\ 355 \omega^{3} M_{0}+O\left(\widetilde{r} \Omega_{2}+N^{2} Q^{-1-\varepsilon_{1}}\right) & \text { if } 0.066<\omega \leq 0.1, \\ 600 \omega^{3} M_{0}+O\left(\widetilde{r} \Omega_{2}+N^{2} Q^{-1-\varepsilon_{1}}\right) & \text { if } 0.006<\omega \leq 0.066, \\ 1680 \omega^{3} M_{0}+O\left(\widetilde{r} \Omega_{2}+N^{2} Q^{-1-\varepsilon_{1}}\right) & \text { if } 0.0025<\omega \leq 0.006, \\ 1791 \omega^{3} M_{0}+O\left(\widetilde{r} \Omega_{2}+N^{2} Q^{-1-\varepsilon_{1}}\right) & \text { if } 10^{-6}<\omega \leq 0.0025, \\ 1876 \omega^{3} M_{0}+O\left(\widetilde{r} \Omega_{2}+N^{2} Q^{-1-\varepsilon_{1}}\right) & \text { if } \omega \leq 10^{-6} .\end{cases}
$$

Proof. By Lemma 1 we have $\omega \leq 0.364$. We only prove the lemma for $0.34<\omega \leq 0.364$. Noting $N_{j}^{\prime} \geq 4^{-1} Q^{38 / 3-20 \varepsilon_{1}-1 / 3}$, as in Lemma 5.5 of [9] we have for $1 \leq j \leq 3$,

$$
1-N_{j}^{\prime \widetilde{\beta}-1} \geq 2.7164 \omega .
$$

Hence the lemma follows.

Lemma 14. We have $M_{1}+M_{3}=M_{0}+O\left(\Omega_{2}+N^{2} \widetilde{r}^{-1} \log ^{3} L+N^{2} Q^{-1-\varepsilon_{1}}\right)$.

This is Lemma 5.6 of [9].

\section{Further estimates on triple sums}

LEMma 15. Let $\varepsilon_{2}$ be a fixed sufficiently small positive constant and $Q>$ $K\left(\varepsilon_{2}\right)$, a positive constant depending on $\varepsilon_{2}$ only. Suppose that the exceptional zero $\widetilde{\beta}$ exists and satisfies $\omega \leq \varepsilon_{2}$. Then for $1 \leq j \leq 3$,

$$
\Sigma_{2}:=\sum_{q \leq Q} \sum_{\chi(\bmod q)}^{*} \sum_{|\gamma| \leq Q^{1+\varepsilon_{1}} q^{-1}}^{\prime} N_{j}^{\beta-1} \ll \varepsilon_{2}^{1 / 2} \omega^{3} .
$$

Proof. Noting that $N_{j} \geq Q^{38 / 3-20 \varepsilon_{1}-1 / 3}$, as in Lemma 6.1 of [9], by Lemmas 3 and 6 we may take

$$
\eta(Q):=\frac{1-\varepsilon_{3}}{(9 / 4) \mathcal{L}} \log \left(\frac{1-\varepsilon_{3}}{(9 / 4) \omega}\right)
$$


where $\varepsilon_{3}=\varepsilon_{3}\left(c, \varepsilon, \varepsilon_{1}\right)$ is a positive constant depending on $c, \varepsilon, \varepsilon_{1}$ only. And $\varepsilon_{3}$ becomes sufficiently small if $c, \varepsilon$ and $\varepsilon_{1}$ are chosen to be sufficiently small. Hence the lemma follows.

LEMma 16. Under the notations of Lemma 15, for any positive constant $C>0$ let $Q>K\left(C, \varepsilon_{2}\right)$, a positive constant depending on $C$ and $\varepsilon_{2}$ only. If (i) $\widetilde{\beta}$ does not exist or (ii) $\widetilde{\beta}$ exists and satisfies $\omega>\varepsilon_{2}$, then we have for $1 \leq j \leq 3$,

$$
\begin{aligned}
\Sigma_{3}: & =\sum_{q \leq Q} \sum_{\chi(\bmod q)}^{*} \sum_{|\gamma| \leq C}^{\prime} N_{j}^{\prime \beta-1} \\
& \leq \begin{cases}0.3511263 & \text { if } \widetilde{\beta} \text { does not exist, } \\
0.083376 & \text { if } 0.306<\omega \leq 0.364, \\
0.0302676 & \text { if } 0.2<\omega \leq 0.306, \\
0.002229681 & \text { if } 0.066<\omega \leq 0.2, \\
0.964773 \cdot 10^{-6} & \text { if } 0.0025<\omega \leq 0.066, \\
0.29853221 \cdot 10^{-16} & \text { if } 10^{-6}<\omega \leq 0.0025, \\
0.001 \omega^{3} & \text { if } \omega \leq 10^{-6} .\end{cases}
\end{aligned}
$$

Proof. We first prove the lemma under the assumption that the exceptional zero $\widetilde{\beta}$ does not exist. By Lemma 1 and in view of the bounds for $\lambda$ in Lemma 2 , we can write

$$
\begin{aligned}
\Sigma_{3}= & N_{j}^{\prime-1 / 2} N^{*}(1 / 2, Q, C) \\
& +\left\{\int_{1 / 2}^{11 / 14}+\int_{11 / 14}^{1-\mathcal{L}^{-1} \log \log \mathcal{L}}+\int_{1-\mathcal{L}^{-1} \log \log \mathcal{L}}^{1-6 / \mathcal{L}}+\int_{1-6 / \mathcal{L}}^{1-2 / \mathcal{L}}+\int_{1-2 / \mathcal{L}}^{1-1 / \mathcal{L}}+\int_{1-1 / \mathcal{L}}^{1-0.696 / \mathcal{L}}\right. \\
& \left.+\int_{1-0.696 / \mathcal{L}}^{1-0.504 / \mathcal{L}}+\int_{1-0.504 / \mathcal{L}}^{1-0.364 / \mathcal{L}}\right\} N^{*}(\alpha, Q, C) N_{j}^{\prime \alpha-1} \log N_{j}^{\prime} d \alpha \\
=: & \sum_{l=1}^{9} C_{l}, \quad \text { say. }
\end{aligned}
$$

As in Lemma 6.2 of [9], by Lemmas 4 and 6 we have

$$
\Sigma_{3} \leq 0.3511263 \text {. }
$$

Now we come to estimating $\Sigma_{3}$ under the assumption that $\widetilde{\beta}$ exists. We separate the arguments in six cases according to the value of the upper bound for $\omega: 10^{-6}, 0.0025,0.066,0.2,0.306$ and 0.364 . We only prove the case $\varepsilon_{2}<\omega \leq 10^{-6}$, other cases can be proved in the same way as in Lemma 6.2 of [9]. If $\omega \leq 10^{-6}$, then by noting $\omega \geq \varepsilon_{2}$, for any zero $\varrho=\beta+i \gamma(\neq \widetilde{\beta})$, we have

$$
\beta \leq 1-\eta(Q)
$$


where $\eta(Q)$ is defined in Lemma 15 . Hence

$$
\begin{aligned}
\Sigma_{3} \leq & (\log \mathcal{L})^{-6} \omega^{3} \\
& +\int_{1-\mathcal{L}^{-1} \log \log \mathcal{L}}^{1-\eta(Q)} N^{*}(\alpha, Q, C) Q^{\left(38 / 3-20 \varepsilon_{1}-1 / 3\right)(\alpha-1)} \log N d \alpha
\end{aligned}
$$

To estimate the integral, we consider two cases according as $\eta(Q) \geq 6 / \mathcal{L}$ or not. When $\eta(Q) \geq 6 / \mathcal{L}$, we get $\omega \leq(4 / 9) \exp (-27 / 2-\varepsilon)$. Thus by Lemma 4 the integral in (5.3) can be estimated as

$$
\begin{aligned}
& \leq \int_{\eta(Q) \mathcal{L}}^{\log \log \mathcal{L}}(38 / 3)(42.54)(1+35.385 / 6) \exp (2.87538 \lambda) Q^{-\left(38 / 3-21 \varepsilon_{1}-1 / 3\right) \lambda \mathcal{L}^{-1}} d \alpha \\
& \leq 394\left(\frac{(9 / 4) \omega}{1-\varepsilon_{3}}\right)^{\left(1-\varepsilon_{3}\right)\left(\left(38 / 3-21 \varepsilon_{1}-1 / 3-2.87538\right) 4\right) / 9} \\
& \leq 394\left(\frac{(9 / 4) \omega}{1-\varepsilon_{3}}\right)^{4.2} \leq 0.0005 \omega^{3}
\end{aligned}
$$

When $\eta(Q) \leq 6 / \mathcal{L}$, we get $\omega>\left(9 / 4+\varepsilon_{3}\right)^{-1} \exp \left(-27 / 2-\varepsilon_{3}\right)$. Thus in view of $C_{4} \leq 0.873 \cdot 10^{-22}$, the integral in (5.3) is

$$
\begin{aligned}
\leq & \frac{0.873 \cdot 10^{-22}}{\left(\left(9 / 4+\varepsilon_{3}\right)^{-1} \exp \left(-27 / 2-\varepsilon_{3}\right)\right)^{3}} \omega^{3} \\
& +\int_{1-6 / \mathcal{L}}^{1-\eta(Q)} N^{*}(\alpha, Q, C) Q^{\left(38 / 3-20 \varepsilon_{1}-1 / 3\right)(\alpha-1)} \log N d \alpha \\
\leq & 0.0004 \omega^{3}+\int_{1-6 / \mathcal{L}}^{1-\eta(Q)} N^{*}(\alpha, Q, C) Q^{\left(38 / 3-20 \varepsilon_{1}-1 / 3\right)(\alpha-1)} \log N d \alpha .
\end{aligned}
$$

By page 374 of $[9]$ we have $\beta \leq 1-4.55 / \mathcal{L}$; by Lemma 4 the last integral in (5.4) is

$$
\begin{aligned}
& \leq \int_{\eta(Q) \mathcal{L}}^{6}(38 / 3)(167.67)(4.55)^{-1} \exp (3.116796 \lambda) Q^{\left(38 / 3-20 \varepsilon_{1}-1 / 3\right)(-\lambda / \mathcal{L})} d \lambda \\
& \leq 52\left(\frac{(9 / 4) \omega}{1-\varepsilon_{3}}\right)^{4.09} \leq 0.0005 \omega^{3} .
\end{aligned}
$$

Hence $\Sigma_{3} \leq 0.001 \omega^{3}$.

LEMMA 17. Under the notations of Lemma 14, for any positive constant $C>0$ let $Q>K\left(C, \varepsilon_{2}\right)$, a positive constant depending on $C$ and $\varepsilon_{2}$ only. Then we have for $1 \leq j \leq 3$, 


$$
\begin{aligned}
& \Sigma_{4}:=\sum_{q \leq B^{1+\varepsilon_{2}}} \sum_{\chi(\bmod q)}^{*} \sum_{|\gamma| \leq C}^{\prime} N_{j}^{\prime \beta-1} \leq 0.366 \cdot 10^{-5}, \\
& \Sigma_{5}:=\sum_{q \leq B^{2+\varepsilon_{2}}} \sum_{\chi(\bmod q)} \sum_{|\gamma| \leq C}^{\prime} N_{j}^{\prime \beta-1} \leq 0.012922 .
\end{aligned}
$$

Proof. This follows in the same way as in the case where the exceptional zero $\widetilde{\beta}$ does not exist in Lemma 16 .

\section{The estimation of $M_{2}$}

LEMMA 18. For any absolute constant $C>1$, we have for $1 \leq j \leq 3$,

$$
\begin{aligned}
S_{2, j} & :=\sum_{q \leq Q} \sum_{\chi(\bmod q)}^{*} \sum_{C \leq|\gamma| \leq T}\left(\int_{-\tau / q}^{\tau / q}\left|\int_{N_{j}^{\prime}}^{N_{j}} x^{\varrho-1} e\left(a_{j} x \eta\right) d x\right|^{3} d \eta\right)^{1 / 3} \\
& \ll C^{-1 / 6} N^{2 / 3}\left|a_{j}\right|^{-1} .
\end{aligned}
$$

Proof. This follows in the same way as Lemma 7.1 of [9].

LEMMA 19. We have for $1 \leq j \leq 3$,

$$
S_{3, j}:=\sum_{q \leq Q} \sum_{\chi(\bmod q)}^{*} \sum_{|\gamma| \leq T}^{\prime}\left(\int_{-\tau / q}^{\tau / q}\left|\int_{N_{j}^{\prime}}^{N_{j}} x^{\varrho-1} e\left(a_{j} x \eta\right) d x\right|^{3} d \eta\right)^{1 / 3} \ll N^{2 / 3}\left|a_{j}\right|^{-1} \text {. }
$$

Proof. Just as in Lemma 7.2 of [9], this follows from Lemma 7.

Lemma 20. For the $\varepsilon_{2}>0$ given as in Lemma 15, let $Q>K\left(\varepsilon_{2}\right)$, a positive constant depending on $\varepsilon_{2}$ only. If $\widetilde{\beta}$ exists and satisfies $\omega \leq$ $\varepsilon_{2}$, then $S_{3, j}$ defined as in Lemma 19 can be estimated further as $S_{3, j} \ll$ $\varepsilon_{2}^{1 / 2} \omega^{3} N^{2 / 3}\left|a_{j}\right|^{-1}$.

Proof. This follows from Lemma 15 as in Lemma 7.3 of [9].

Now we come to estimating $M_{2}$. We consider two cases according as the exceptional zero $\widetilde{\beta}$ exists or not.

If $\widetilde{\beta}$ does not exist, then there are 7 terms in the integrand of $M_{2}$ and they are the first, fourth and sixth type in case (I) of p. 376 of [9]. For $M_{21}$, as for $M_{25}$ of [9], we get

$$
\begin{aligned}
M_{21}= & \sum_{r_{3} \leq Q} \sum_{\chi_{3}}^{*} \sum_{\substack{\bmod r_{3} \\
\left|\gamma_{3}\right| \leq C}}^{\prime} \sum_{\substack{q \leq Q \\
r_{3} \mid q}} \varphi^{-3}(q) Z\left(q ; \chi_{0}, \chi_{0}, \bar{\chi}_{3}\right) \\
& \times \int_{-\infty}^{\infty} e(-b \eta)\left(\prod_{j=1}^{2} \int_{N_{j}^{\prime}}^{N_{j}} e\left(a_{j} x \eta\right) d x\right)\left(\int_{N_{3}^{\prime}}^{N_{3}} x^{\varrho_{3}-1} e\left(a_{3} x \eta\right) d x\right) d \eta \\
& +O\left(N^{2}\left|a_{1} a_{2} a_{3}\right|^{-1} C^{-1 / 6} \prod_{p} s(p)\right) .
\end{aligned}
$$


By Lemma 11 we get

$$
\begin{aligned}
M_{21}= & \sum_{r_{3} \leq B^{1+2 \varepsilon_{1}}} \sum_{\chi_{3}\left(\bmod r_{3}\right)}^{*} \sum_{\left|\gamma_{3}\right| \leq C}^{\prime} \sum_{\substack{q \leq Q \\
r_{3} \mid q}} \varphi^{-3}(q) Z\left(q ; \chi_{0}, \chi_{0}, \bar{\chi}_{3}\right) \\
& \times \int_{-\infty}^{\infty} e(-b \eta)\left(\prod_{j=1}^{2} \int_{N_{j}^{\prime}}^{N_{j}} e\left(a_{j} x \eta\right) d x\right)\left(\int_{N_{3}^{\prime}}^{N_{3}} x^{\varrho_{3}-1} e\left(a_{3} x \eta\right) d x\right) d \eta \\
& +O\left(N^{2}\left|a_{1} a_{2} a_{3}\right|^{-1} C^{-1 / 6} \prod_{p} s(p)\right)+O\left(N^{2}\left|a_{1} a_{2} a_{3}\right|^{-1} Q^{-\varepsilon_{1}} \prod_{p} s(p)\right) .
\end{aligned}
$$

Hence we have

$$
\begin{aligned}
\left|M_{21}\right| \leq & N^{2}\left|a_{3}\right|^{-1}(2.140782)\left(\Sigma_{4}\right)\left(\prod_{p} s(p)\right) \int_{\mathcal{D}} d x_{1} d x_{2} \\
& +o\left(N^{2}\left|a_{1} a_{2} a_{3}\right|^{-1} \prod_{p} s(p)\right) \\
\leq & (2.140782+\varepsilon)\left(\Sigma_{4}\right) M_{0} .
\end{aligned}
$$

Similarly, we have

$$
\begin{aligned}
& \left|M_{24}\right| \leq(2.140782+\varepsilon)\left(\Sigma_{5}\right)^{2} M_{0}, \\
& \left|M_{26}\right| \leq(2.140782+\varepsilon)\left(\Sigma_{3}\right)^{3} M_{0},
\end{aligned}
$$

providing that $C$ is large enough. Consequently,

$$
\begin{aligned}
M_{2} \leq & (2.140782+\varepsilon) \\
& \times\left(3\left(0.366 \cdot 10^{-5}\right)+3(0.012922)^{2}+(0.3511263)^{3}\right) M_{0} \\
\leq & 0.096 M_{0} .
\end{aligned}
$$

If the exceptional zero exists, then as on page 376 of [9], there are 19 terms in the integrand of $M_{2}$ and they are of 6 types; the treatment of these six types is quite similar to be above, so we have

$$
\begin{aligned}
& \left|M_{21}\right| \leq(2.140782+\varepsilon)\left(\Sigma_{4}\right) M_{0}, \\
& \left|M_{22}\right| \leq(2.140782+\varepsilon)\left(\Sigma_{5}\right) M_{0}, \\
& \left|M_{23}\right| \leq(2.140782+\varepsilon)\left(\Sigma_{3}\right) M_{0}, \\
& \left|M_{24}\right| \leq(2.140782+\varepsilon)\left(\Sigma_{5}\right)^{2} M_{0}, \\
& \left|M_{25}\right| \leq(2.140782+\varepsilon)\left(\Sigma_{3}\right)^{2} M_{0}, \\
& \left|M_{26}\right| \leq(2.140782+\varepsilon)\left(\Sigma_{3}\right)^{3} M_{0},
\end{aligned}
$$

(6.2) $\quad M_{2} \leq(2.140782+\varepsilon)$

$$
\times\left(3\left(\Sigma_{4}\right)+6\left(\Sigma_{5}\right)+3\left(\Sigma_{3}\right)+3\left(\Sigma_{5}\right)^{2}+3\left(\Sigma_{3}\right)^{2}+\left(\Sigma_{3}\right)^{3}\right) M_{0} .
$$


Note that trivially we have

$$
\Sigma_{4} \leq \Sigma_{5} \leq \Sigma_{3}
$$

By Lemmas 16 and 17 one can deduce the following.

Lemma 21. For the $\varepsilon_{2}>0$ given as in Lemma 15, let $Q>K\left(\varepsilon_{2}\right)$, a positive constant depending on $\varepsilon_{2}$ only. Under the assumptions in Lemma 12, we have

$$
M_{2} \leq \begin{cases}0.096 & \text { if } \widetilde{\beta} \text { does not exist } \\ 0.749 & \text { if } 0.306<\omega \leq 0.364 \\ 0.368 & \text { if } 0.2<\omega \leq 0.306 \\ 0.0431 & \text { if } 0.066<\omega \leq 0.2 \\ 0.2479 \cdot 10^{-4} & \text { if } 0.0025<\omega \leq 0.066 \\ 0.767 \cdot 10^{-15} & \text { if } 10^{-6}<\omega \leq 0.0025 \\ 0.05 \omega^{3} & \text { if } \varepsilon_{2}<\omega \leq 10^{-6}\end{cases}
$$

\section{Proof of Theorem 1}

Lemma 22. Let $I_{2}(b)$ be defined as in (3.7). Then

$$
I_{2}(b) \ll N^{2} Q^{-1 / 2}\left|a_{1} a_{2} a_{3}\right|^{-1 / 2} L^{5} .
$$

This is Lemma 8.1 of $[9]$.

LEMMA 23. Let $I_{1}(b)$ be defined as in (3.7) and $\theta$ be given as in (3.1). Under the assumptions of Lemma 12 and (3.4), we have $I_{1}(b) \gg \omega^{3} M_{0}$.

Proof. Just as in Lemma 8.2 of [9], this follows from Lemmas 9, 12, 13, 14,21 , and (4.2).

Proof of Theorem 1. By (3.7), Lemmas 22, 23, 12, and noting $\omega \geq Q^{-\varepsilon}$, we get

$$
I(b)=I_{1}(b)+I_{2}(b) \gg \omega^{3} N^{2}\left|a_{1} a_{2} a_{3}\right|^{-1}
$$

providing that $Q \gg B^{3+3 \varepsilon_{1}}$, that is, $N \gg B^{\left(3+3 \varepsilon_{1}\right)\left(38 / 3-20 \varepsilon_{1}\right)} \gg B^{38-22 \varepsilon_{1}-60 \varepsilon_{1}^{2}}$ with $\varepsilon_{1}$ small enough. Noting the assumptions of Lemma 12, the proof of Theorem 1 is complete.

\section{References}

[1] A. Baker, On some diophantine inequalities involving primes, J. Reine Angew. Math. 228 (1967), 166-181.

[2] K. K. Choi, A numerical bound for Baker's constant-some explicit estimates for small prime solutions of linear equations, Bull. Hong Kong Math. Soc. 1 (1997), $1-19$.

[3] K. K. Choi, M. C. Liu and K. M. Tsang, Conditional bounds for small prime solutions of linear equations, Manuscripta Math. 74 (1992), 321-340. 
[4] D. R. Heath-Brown, The density of zeros of Dirichlet's L-functions, Canad. J. Math. 31 (1979), 231-240.

[5] - Zero-free regions for Dirichet L-functions, and the least prime in an arithmetic progression, Proc. London Math. Soc. 64 (1992), 265-338.

[6] H. Z. Li, Zero-free regions for Dirichlet L-functions, Quart. J. Math. Oxford Ser. 50 (1999), 13-23.

[7] M. C. Liu and K. M. Tsang, Small prime solutions of linear equations, in: Théorie des Nombres, J.-M. De Koninck and C. Levesque (eds.), de Gruyter, 1989, 595-624.

[8] —, 一, Recent progress on a problem of A. Baker, in: Séminaire de Théorie des Nombres, Paris 1991-1992, Progr. Math. 116, Birkhäuser, 1993, 121-133.

[9] M. C. Liu and T. Z. Wang, A numerical bound for small prime solutions of some ternary linear equations, Acta Arith. 86 (1998), 343-383.

[10] C. D. Pan and C. B. Pan, Goldbach Conjecture, Science Press, Beijing, 1992.

Department of Mathematics

Shandong University

Jinan, Shandong 250100

P.R. China

E-mail: lihz@sdu.edu.cn

Received on 23.3.2000

and in revised form on 3.8.2000 